Dissertation Abstract

\title{
Effectiveness of individualized homeopathic treatment in perennial allergic rhinitis (PAR)
}

\author{
Marcus Zulian Teixeira \\ Milton de Arruda Martins (advisor) \\ Universidade de São Paulo, São Paulo, Brazil
}

\begin{abstract}
Homeopathic therapy was formulated in 1796 by German physician Samuel Hahnemann aiming at stimulating the vital reaction of the organism against its own disturbs. The scientific rationality of homeopathy presents four main suppositions: principle of therapeutic similarity, experimentation of medicinal substances on healthy individuals, potentized medicines and individualized medicines. Shortly, homeopathy prescribes to sick individuals remedies that have caused similar symptoms in healthy individuals in infinitesimal doses (in order to avoid the initial aggravation of symptoms) by taking into account the varied individual traits of patients (mental, general and local symptoms) in the choice of the most suited remedy (individualized medicine).
\end{abstract}

By using a global approach to semiology aimed at discovering the totality of symptoms and signs characteristic for a particular pair patient-disease, homeopathic physicians make some medicinal hypotheses among the thousands of tested substances which must be prescribed one by one gradually with periodical reevaluations until the desired healing reaction is achieved (middle-to-long-term treatment).

These particularities of the homeopathic model require specific attention when designing scientific research protocols as they differ significantly from the standards employed by clinical epidemiology. To begin with, a relative long period of time is needed in randomized controlled double-blind trials (RCTs) in order to find the suitable individualized remedies, which are the cornerstone for awakening a global homeostatic reaction and to alter the natural course of chronic diseases, which is the ideal aim of homeopathic cure.

This doctoral thesis proposes a model for clinical trials complying with the standards of both conventional and homeopathic medicine. Taking into account the high rated of drop-outs in long-term RCTs in both cases, which makes traditional quantitative evaluation impossible, a "mixed" RCT was designed, including an initial closed phase (randomized, controlled, double-blind: Phase 1) lasting 6 months and a later open phase (Phase 2) lasting 12-36 months using exclusively homeopathic treatment, with quantitative and qualitative analyses all along the study (while taking into account the possible systematic error in this kind of clinical trial). This design, besides allowing the analysis of non specific data in homeopathic treatment (placebo effect, patientdoctor relationship, etc.) favors the restriction of placebo to 6 months, complying thus with ethical regulations for clinical research.

Patients were assessed by a physician accredited in allergy and immunology nominated by the Department of Allergy and Clinical Immunology of FMUSP hospital, who had no direct association with this study and employed the Department's standard "Allergic Rhinitis Form", a score composed of the specific signs and symptoms of allergic rhinitis and the main outcome of treatments. 
Study population comprised adults (average age $=30$ year-old) with moderate allergic rhinitis (average score $=15 / 24)$ an average duration of disease $=14$ years in habitual and long-term use of conventional treatments without any alteration in the natural course of the disease.

From 55 randomized patients, 41 completed Phase 1; 21 in the group with homeopathic treatment and 20 in the group treated with placebo; there was average 25\% improvement compared with the baseline score without significant difference between both groups.

In phase 2 about $70 \%$ of patents $(\mathrm{n}=27)$ completed 12 months of individualized homeopathic treatment and present $50 \%$ improvement regarding baseline clinical score significant by comparison to improvement in Phase 1; 50\% $(\mathrm{n}=20)$ and $30 \%(\mathrm{n}=13)$ patients completed 24 and 36 months of homeopathic treatment respectively, with $64 \%$ and $72 \%$ of improvement regarding baseline clinical score, both significant by comparison to improvement in Phase 1. (Figure 1)

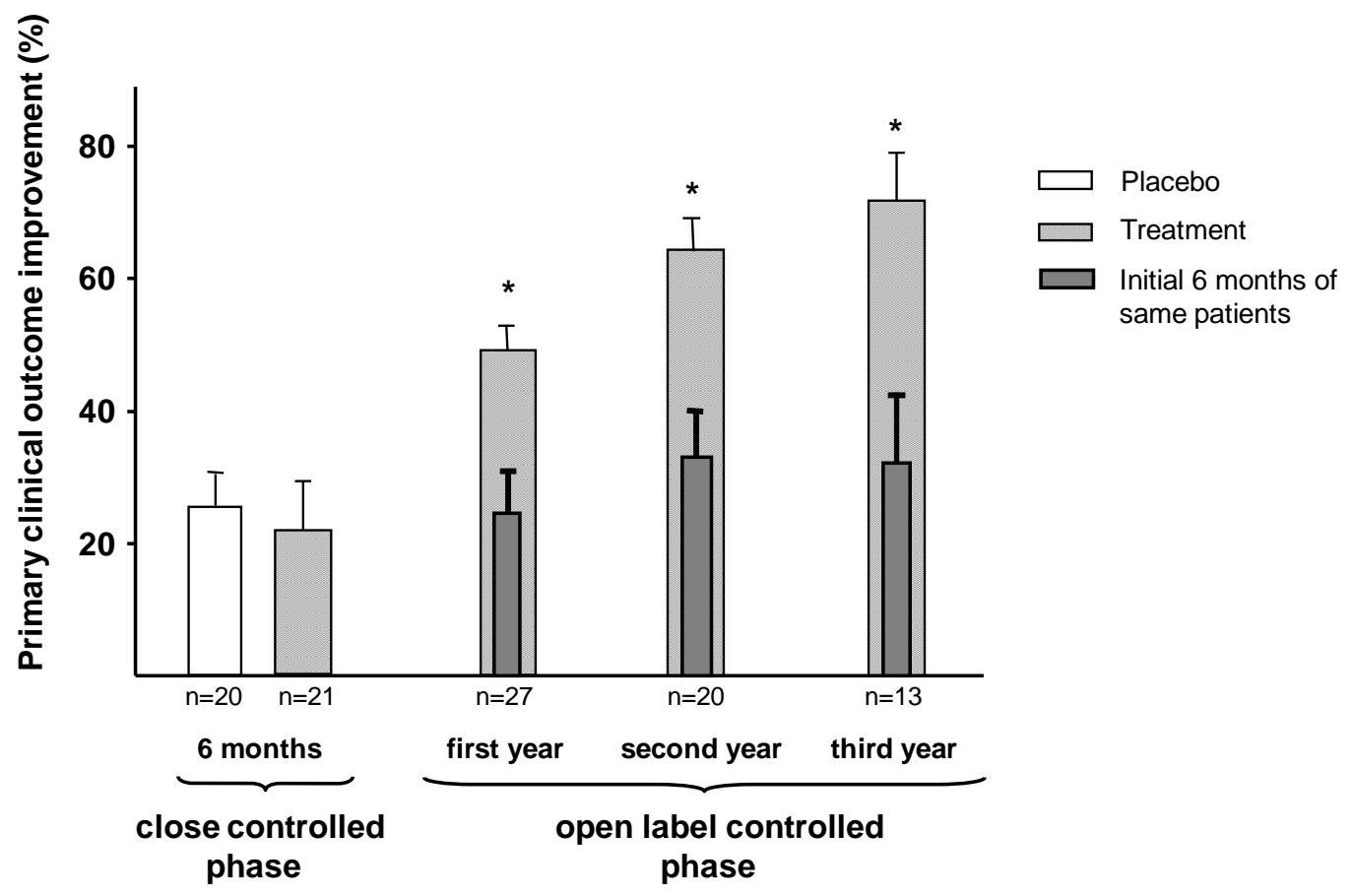

Figure 1. Improvement percentage of allergic rhinitis specific symptoms and signs score (primary clinical outcome) during first (closed controlled phase) and second phase (open label controlled phase). [*] $\mathrm{p}<0.05$ when compared with closed controlled phase (initial six months) of same patients.

After suspension of homeopathic treatment, a qualitative analysis showed that patients who reached $100 \%$ improvement at some point in the course of the study (correctly identified remedy) remained average 3 years without any sign or symptom, indicating a change in the natural course of the disease. 
Possible systematic error (bias in selection of patients, measurement and confusion of results) were listed and discussed along the study. The use of the same evaluation tool applied by the same immunologist doctor in all phases of the study rules out bias in measurement of results. It was neither observed bias in selection of patients (non respondent to treatment or less chronicity of disease) in Phase 2, whereas 85\% of drop-outs adduced as main reason a substantial improvement of the original allergic illness; patients dropping out before completing 12 months of homeopathic treatment presented decreased chronicity of the disease.

Regarding bias of confusion of results in Phase 2, where unspecific results of treatment can be confounded with the specific results of homeopathic remedies (principle of therapeutic similarity) analyses of the evolution of both initial subgroups (treatment and placebo) sought to minimize these questionings. By comparison to improvement in Phase 1, the subgroup of patients coming from the initial placebo group ( $\mathrm{n}=20)$ showed significant improvement only at the end of the same 12 months of homeopathic treatment (6 months Phase $1+12$ months Phase 2) without significant difference at the end of the initial 12 months of unspecific effects received (6 months Phase $1+6$ months Phase 2). Other qualitative and individual analyses of patients, where placebo and nocebo effects were confronted, strengthened these interpretations.

With the experience acquired along this long-term homeopathic clinical trial, the author discusses new proposals and suggestions to improve the quality of future studies in order to increase knowledge on the efficacy of homeopathic treatment in human diseases.

\section{Reference:}

Teixeira MZ. Ensaio clínico quali-quantitativo para avaliar a eficácia e a efetividade do tratamento homeopático individualizado na rinite alérgica perene [dissertation online]. São Paulo (Brazil): Faculdade de Medicina, Universidade de São Paulo; 2009. 3.47MB. Available at http://www.teses.usp.br/teses/disponiveis/5/5159/tde-10062009-102220/. [portuguese].

\section{(c)) BY-NC-ND Licensed to GIRI}

Support: authors declare that this study received no funding

Conflict of interest: authors declare there is no conflict of interest

Received: 15 September 2009; Revised 23 September 2009; Published: 30 September 2009

Correspondence author: Marcus Zulian Teixeira, marcus@homeozulian.med.br, www.homeozulian.med.br.

How to cite this article: Teixeira MZ. Effectiveness of individualized homeopathic treatment in perennial allergic rhinitis (PAR) [dissertation abstract]. Int J High Dilution Res. 2009 [cited YYYY Month dd]; 8 (28): 141-143. Available from: http://www.feg.unesp.br/ ojs/index.php/ijhdr/article/view/351/403. 